Cahiers québécois de démographie

Revue internationale d'étude des populations

\title{
La démographie linguistique : un chantier de recherche qui s’internationalise
}

\section{Richard Marcoux et Moussa Bougma}

Volume 46, numéro 2, automne 2017

Dynamiques linguistiques

URI : https://id.erudit.org/iderudit/1054051ar

DOI : https://doi.org/10.7202/1054051ar

Aller au sommaire du numéro

Éditeur(s)

Association des démographes du Québec

ISSN

1705-1495 (numérique)

Découvrir la revue

Citer ce document

Marcoux, R. \& Bougma, M. (2017). La démographie linguistique : un chantier de recherche qui s'internationalise. Cahiers québécois de démographie, 46(2),

189-196. https://doi.org/10.7202/1054051ar d'utilisation que vous pouvez consulter en ligne. 


\title{
Cahiers québécois de démographie
}

Vol. 46, n 2, automne 2017, p. 189-196

\section{Introduction La démographie linguistique : un chantier de recherche qui s'internationalise}

\author{
RICHARD MARCOUX ET MOUSSA BOUGMA*
}

$I^{1}$ ly a maintenant plus de trente ans, feu Robert Maheu pilotait un excellent numéro des Cahiers québécois de démographie avec pour titre un simple mot : "Démolinguistique ". Ce numéro, paru en 1985, révélait bien l'immense chantier de recherche dans lequel s’inscrivaient déjà plusieurs démographes du Québec.

«La démolinguistique est une branche de la démographie. C'est l'étude des populations caractérisées par une appartenance à une langue commune. Le plus souvent les études de démolinguistique visent à comparer les comportements démographiques de groupes linguistiques vivant sur un même territoire. Cela donne à la démolinguistique une dimension politique indéniable. C'était le cas en Belgique lorsqu'on y tenait des recensements linguistiques. C'est le cas au Québec et au Canada où les questions linguistiques font l'objet d'interventions des administrateurs publiques.» (Maheu, 1985: p. 3).

Il n'est pas étonnant de constater que cette «branche de la démographie » puisse occuper une place aussi importante au Québec. Les débats entourant l'avenir de la langue française y sont récurrents et traversent, avec des intensités variables, l'histoire complexe du Québec et du Canada depuis près de deux siècles (Piché, 2011). Les affrontements se sont assurément exacerbés au cours des soixante dernières années, suivant la mise en place de différentes commissions fédérale et provinciale qui ont, pour la plupart, conduit à l'adoption de lois et de réglementations sur les langues ${ }^{1}$. Les démographes du Québec ont souvent été " appelés à la barre " afin de contribuer à

* Richard Marcoux est professeur titulaire et directeur de l'Observatoire démographique et statistique de l'espace francophone (ODSEF) à l'Université Laval. Moussa Bougma est maître-assistant en démographie à l'Institut supérieur des sciences de la population (ISSP) de l'Université Ouaga I Professeur Joseph Ki-Zerbo.

1. Soulignons quelques exemples: Commission royale d'enquête sur le bilinguisme et le biculturalisme (Commission Laurendeau-Dunton) au milieu des années 1960; Commission d'enquête sur la situation de la langue française et sur les droits linguistiques au Québec (dite Commission Gendron) durant la fin des années 1960 et au début 1970 et qui a conduit à l'adoption de la loi sur la langue officielle (loi 22) en 1974 et à la Charte de la langue française adoptée en 1977. Rappelons aussi plus récemment la tenue de la Commission des états généraux sur la situation et l'avenir de la langue française au Québec (Commission Larose) en 2000-2001; et plus récemment, les débats entourant la "Charte des valeurs québécoises » 
ces débats, situant leurs conclusions dans un spectre assez large concernant l'évolution future des francophones au Québec et au Canada ; certains oscillant même entre les visions optimistes et pessimistes au cours de leur carrière, comme l'a si bien illustré Marc Termote (2015) concernant l'évolution du discours démolinguistique de Jacques Henripin.

Tous ces débats ont conduit différentes agences gouvernementales à collecter de plus en plus d'informations sur les langues, faisant du Québec un lieu particulièrement privilégié et intellectuellement stimulant pour l'étude des dynamiques linguistiques. Ainsi, avant le milieu des années soixante, les questionnaires des recensements canadiens s'intéressaient uniquement aux langues maternelles et à la maîtrise des deux langues officielles au Canada (Houle et Cambron-Prémont, 2015). Dès 1971, Statistique Canada ajoute de nouvelles questions dans le questionnaire du recensement, notamment sur la langue parlée à la maison. Depuis le recensement de 2001, on compte jusqu'à sept principales questions distinctes de nature linguistique dans les différents questionnaires, y compris sur les langues de travail (Lachapelle, 2008).

Outre les recensements, de nombreuses enquêtes spécifiques sur les langues ont été menées sous la direction de l'agence canadienne de statistique, mais également par l'Office québécois de la langue française (OQLF) et d'autres organismes. Une variable sur la langue est d'ailleurs toujours présente dans les enquêtes d'opinion menées par les maisons de sondage au Québec: on ne peut imaginer la publication des intentions de votes sans tenir compte des distinctions entre francophones et anglophones. En somme, le Canada et le Québec sont un peu présentés comme l'eldorado des données démolinguistiques. L'important inventaire des sources permettant d'identifier les francophones dans le monde, réalisé par Bruno Maurer (2015), illustre bien la quantité et la qualité des données accessibles aux chercheurs qui s'intéressent à la langue française au Québec et au Canada. La vaste revue de littérature internationale produite sous le titre "Compter les langues » donne une place centrale au Canada et au Québec dans la production de connaissance dans le domaine de la démolinguistique. (Humbert, Coray et Duchêne, 2018)

On peut d'ailleurs s'attendre à un élargissement encore plus grand des intérêts pour les langues. À la suite des excuses officielles du gouvernement canadien, présentées en 2008 aux peuples autochtones pour les mauvais traitements et sévices subis dans les pensionnats, on observe un intérêt grandissant concernant la survie des langues des populations amérindiennes et des Inuits (Guimond et Sénécal, 2009; Sioui Durand, 2018). Il s'agit assurément d'un axe de recherche amené à se développer, d'autant plus que le gouvernement du Québec a fait de la vitalité des langues autochtones un engagement particulier de sa toute récente politique culturelle (Gouvernement du Québec, 2018).

Bref, suivant ces derniers exemples, on peut assurément confirmer les propos de Robert Maheu (1985) dans la citation plus haut, à savoir que le champ de la démolinguistique comporte indéniablement une dimension politique. Si le Québec et le Canada peuvent représenter des terrains d'études particulièrement riches pour ces travaux, d'autres pays francophones, comme la Belgique et la Suisse, ont aussi fait l'objet d'études fort intéressantes (Prévost et Beaud, 2002 ; Grin, 2010). Dans un autre numéro des Cahiers québécois de démographie, portant cette fois sur les substitu-

(projet de loi 60) et enfin ceux entourant l'idée d'un test de français pour les immigrants tel que proposé par l'un des partis politiques aux élections d'octobre 2018. 
tions linguistiques, Alain Bélanger et Patrick Sabourin (2011) insistent eux aussi sur l'intérêt des études pour d'autres territoires de dualité linguistique. Deux articles de ce numéro qu'ils ont dirigé portent d'ailleurs sur les Communautés autonomes espagnoles.

Devant ces exemples, on peut s'étonner que très peu de pays du Sud, d'Afrique notamment, qui présentent pourtant des contextes de dualité ou de pluralité linguistique fort intéressants, aient si peu suscité l'intérêt des démographes. Les attributs ethnolinguistiques sont pourtant au cœur de nombreux processus de stratification sociale en Afrique. "Le pouvoir aux zarmas et le commerce aux haoussas ", une expression qui illustre l'opposition entre les deux plus importants groupes linguistiques du Niger et que nous rappelle l'auteur d'une thèse de doctorat sur ce pays (Beidou, 2014: p. 261).

Pour ouvrir un chantier de recherche sur la démolinguistique en Afrique il nous fallait remplir deux conditions. Nous devions d'abord vérifier que des sources d'informations démographiques sur les langues existaient en Afrique, ce que nous avons fait avec notre collègue Mamadou Kani Konaté (Marcoux et Konaté, 2008) et que nous avons poursuivi par la suite (Marcoux, Richard et Konaté, 2013 Marcoux et Alladatin, 2014). Il fallait également susciter l'intérêt politique d'un tel chantier, ce que nous avons également relevé en soulignant, sur plusieurs tribunes, le rôle du continent africain concernant l'avenir démographique de la Francophonie (Marcoux, 2003 et 2018 ; Bougma, Konaté et Marcoux, 2010; Marcoux et Konaté, 2012; Marcoux et Wolff, 2014; Marcoux et Richard 2017).

Créé en 2009, l'Observatoire démographique et statistique de l'espace francophone (ODSEF) a notamment contribué à ouvrir ce chantier de recherche. Un des objectifs de l'ODSEF est d'appuyer l'ensemble des initiatives permettant de circonscrire les dynamiques linguistiques et de mieux situer la place qu'occupe la langue française au sein des populations de la Francophonie et d'ailleurs. Des études sur les dynamiques linguistiques ont ainsi pu être produites à l'échelle de la planète (Marcoux, 2018; Marcoux et Richard, 2017) mais plus particulièrement sur de nombreux pays d'Afrique, notamment le Burkina Faso (Bougma,2010), le Mali (Konaté, Diabaté et Assima, 2010), la Tunisie (Bouhdiba, 2011), le Cameroun (Tanang Tchouala et Efon Etinzoh, 2013), le Niger (Ousmane Ida, 2015 et Issaka Maga, 2014) le Rwanda (Uwayezu, 2015) et la Mauritanie (Ba et Echraf Ouédraogo, 2018).

Les articles regroupés dans le présent numéro des Cahiers québécois de démographie mettent de l'avant des contextes qui ont en commun le fait que plusieurs des populations étudiées semblent utiliser plus d'une langue au quotidien. Outre cette expansion du plurilinguisme, ce numéro illustre aussi le caractère international des travaux en cours autour des enjeux démolinguistiques : deux articles portant sur l'Afrique, un autre sur des départements français d'outre-mer et un quatrième qui nous permet d'examiner les plus récentes données tirées du recensement canadien de 2016.

\section{À PROPOS DES ARTICLES DE CE NUMÉRO}

Des informations sur les langues d'alphabétisation sont régulièrement collectées dans les recensements en Afrique, et ce, afin de calculer les taux d'alphabétisation. Compte tenu de l'omniprésence des langues issues de la colonisation dans les programmes d'enseignement de plusieurs pays 
africains, ces informations sont notamment utiles pour nos travaux sur l'estimation des francophones en Afrique (Bougma, Konaté et Marcoux, 2010 ; Marcoux et Alladatin, 2014). Des questions sur les langues parlées y sont toutefois rarement collectées avec un minimum de précisions. À ce titre, les trois derniers recensements généraux de la population du Sénégal (1988, 2002, 2013) constituent une exception, car ceux-ci contiennent des informations sur la première langue et la deuxième langue parlées par chaque membre du ménage au moment du recensement. En exploitant cette richesse d'informations, Diouf et ses collègues s'interrogent sur la dynamique des langues parlées ainsi que leur transmission au sein des ménages sénégalais au cours des 25 dernières années. L'analyse révèle que le paysage linguistique sénégalais est marqué par la prédominance et l'expansion progressive du wolof. À côté de cette langue omniprésente, on retrouve le pulaar et le sérère qui connaissent une dynamique globalement constante malgré une diminution des locuteurs en première langue (pour le sérère) et une expansion du français comme deuxième langue. Cependant, les auteurs soulignent que cette suprématie du wolof et l'expansion du français comme deuxième langue n'empêchent pas pour autant la survie des langues minoritaires telles que le diola, le mandingue et le soninké, comme nous l'avions déjà observé au Sénégal et ailleurs en Afrique (Ouédraogo et Marcoux, 2014). Par conséquent, les auteurs concluent que l'hypothèse d'une érosion des langues nationales au profit du wolof et du français n'est pas encore confirmée dans le contexte sénégalais, et ce, même si l'analyse de la transmission des langues au sein du noyau familial présage, selon eux, une augmentation des locuteurs wolofs par rapport aux autres langues dans les générations futures.

En comparant l'appartenance ethnique et la principale langue parlée au sein du ménage à partir du tout dernier recensement général de la population du Bénin, celui de 2013 où la question sur la principale langue parlée par chacun des membres du ménage a été introduite pour la première fois, Mouftaou Amadou Sani tente de répondre aux interrogations suivantes : l'assimilation linguistique ou l'abandon de la langue maternelle au profit d'une nouvelle langue a-t-elle cours dans les sociétés béninoises? Si oui, quelles sont sa fréquence et ses variations? A-t-elle une influence sur la dimension démo-linguistique des groupes socio-ethniques du Bénin? Les résultats de l'analyse amènent l'auteur à répondre par l'affirmative. Non seulement il démontre que l'assimilation linguistique n'épargne aucune des langues nationales du Bénin, mais il constate aussi que cette assimilation est plus fréquente au fur et à mesure que l'on s'éloigne des aires géographiques béninoises de concentration de chacun des groupes ethniques, particulièrement, dans les agglomérations urbaines et parmi les personnes n'appartenant pas au groupe ethnique du chef de ménage. Le français se trouve être la première langue d'assimilation parmi les personnes non apparentées au chef de ménage et plus globalement, dans les milieux familiaux où cohabitent deux ou plusieurs groupes ethniques.

En exploitant les informations issues de l'enquête française Migrations-Famille-Vieillissement sur les langues reçues à l'enfance et les langues parlées avec les enfants, Baptiste Beck se propose de décrire l'évolution de la transmission du français et du créole - deux langues qui se côtoient dans cet espace depuis plusieurs siècles- dans quatre départements français d'outre-mer : deux situés dans les Caraïbes (Guadeloupe et Martinique), un situé à l'ouest de l'océan indien (La Réunion) et le dernier en Amérique du Sud (Guyane). Dressant d'abord le bilan du bagage linguistique reçu pendant l'enfance et susceptible d'être transmis aux enfants, l'auteur relève que l'enfance des indi- 
vidus se caractérise globalement par une forte présence du créole, et ce, dans chacun des départements. En Martinique et en Guadeloupe, l'éducation en créole par les parents se fait en parallèle à l'éducation en français, alors qu'à La Réunion, les éducations bilingues sont moins fréquentes. L'analyse faite par la suite sur la transmission linguistique montre que les générations plus récentes transmettent moins le créole que les générations plus anciennes, et ce, dans l'ensemble des départements, à l'exception de la Réunion où l'auteur constate que, bien qu'en recul, la pratique de la langue créole demeure importante dans les échanges entre les parents et les enfants parmi les générations récentes. L'auteur souligne par ailleurs que dans la plupart des départements, le recul du créole, observé au sein du noyau familial au fil des générations, s'opère au profit de la langue française. Pour l'auteur, cette substitution se fait plutôt sur un cycle de deux générations : les parents unilingues créolophones à l'enfance complexifient le bagage transmis à leurs enfants en y ajoutant le français, et ces enfants à leur tour, une fois devenus parents, simplifient le bagage linguistique en ne conservant que le français.

Le texte de Jean-François Lepage nous permet de revenir au Canada. Utilisant les données des derniers recensements généraux de la population, l'auteur présente quelques éléments de l'évolution récente de la «complexification » du paysage linguistique canadien à l'issue desquels il examine la place que prennent les deux langues officielles dans cet environnement linguistique en mutation, tenant compte de facteurs tels que l'exogamie, l'usage des langues au foyer et la transmission des langues aux enfants. Ses analyses font ressortir une diversification linguistique avec une croissance des «situations complexes » dans l'utilisation des langues au foyer. Les analyses révèlent en effet que la proportion de la population qui n'utilise que sa langue maternelle à la maison a diminué entre 2001 et 2016, et ce, dans un contexte de croissance des langues d'usage tierces (autres que française ou anglaise). Observant au cours de la même période un fort pouvoir d'attraction de la langue majoritaire, particulièrement de l'anglais, Jean-François Lepage explique cette « complexification » du portrait linguistique familial canadien par le fait de l'ajout d'une langue officielle, principalement la langue officielle majoritaire. Ces résultats l'amènent à conclure que malgré la croissance soutenue des langues immigrantes au Canada, la pénétration des langues officielles est importante et contribue au plurilinguisme dans l'espace familial.

\section{À LA MÉMOIRE DE RÉJEAN LACHAPELLE}

Il est intéressant de dresser la liste des auteurs ayant contribué en 1985 à ce numéro du volume 14 des Cahiers québécois de démographie dirigé par Robert Maheux et auquel nous faisions référence au début de ce texte. Ceux-ci font assurément partie des principaux chercheurs ayant contribué au développement de la démoliguistique au Québec et on y retrouve, par ordre d'apparition des articles: Marc Tremblay et Robert Bourbeau, Marc Termote et Danielle Gauvreau, Charles Castonguay, Jacques Henripin, Calvin Veltman et enfin, Réjean Lachapelle.

«Le père de la démolinguistique n'existe pas. Et s'il existait, il faudrait alors aussi chercher la mère ", nous avait lancé en 2012 Réjean Lachapelle, avec son sens de l'humour incomparable. Réjean est venu passer six semaines à Québec au courant de l'été 2012. Il a aussi participé au $1^{\text {er }}$ Forum mondial sur la langue française et à de nombreuses activités organisées par l'ODSEF dans ce cadre. 
Surtout, Réjean nous a accompagné dans l'encadrement des nombreux analystes et chercheurs qui bénéficiaient à l'été 2012 du programme annuel d'accueil de l'ODSEF. Par sa grande érudition accompagnée d'une grande simplicité, Réjean a laissé un souvenir impérissable auprès des analystes et chercheurs en accueil mais aussi auprès de toute l'équipe de l'ODSEF. Il nous a notamment transmis sa passion pour la recherche et pour l'analyse démographique. Il a aussi largement fait état de ses expériences durant sa carrière à Statistique Canada et comment il avait tenté de s'assurer que les opérations de collecte de données menées par cette institution puissent permettre de fournir des éléments de réponse aux questions soulevées autour des enjeux linguistiques. Je soupçonne d'ailleurs que les nouvelles questions sur les langues qui ont été ajoutées dans les récents recensements africains l'ont été suite à ces belles rencontres durant l'été 2012. Bref, Réjean aura assurément marqué le milieu scientifique de la démographie au-delà des frontières du Québec et du Canada.

En accord avec la direction des Cahiers québécois de démographie, nous dédions ce numéro à la mémoire de Réjean Lachapelle qui nous a quittés en mai 2018.

\section{BIBLIOGRAPHIE}

BA, A. Y. et A. ECHRAF OUEDRAOGO. 2018. Portrait des dynamiques linguistiques et de l'alphabétisation en Mauritanie: Une analyse à partir des données du recensement de 2013. Québec, Observatoire démographique et statistique de l'espace francophone, Université Laval, 44 p.

BEIDOU, H. 2014. Dynamiques linguistiques au Niger. Cohabitation et/ou concurrence entre le français et les langues locales, Thèse de doctorat, Montréal, UQAM, 275 p.

BÉLANGER, A. et P. SABOURIN. 2011. " Mesure et déterminants des substitutions linguistiques », Cahiers québécois de démographie, 40(1), 5-12.

BOUGMA, M. 2010. Dynamique des langues locales et de la langue française au Burkina Faso: un éclairage à travers les recensements généraux de la population (1985, 1996 et 2006). Québec : Observatoire démographique et statistique de l'espace francophone / Université Laval, $61 \mathrm{p}$.

BOUGMA, M., M. K. KONATÉ et R. MARCOUX. 2010. "Les populations francophones au Burkina et au Mali depuis le milieu des années 1980 », dans La langue française dans le monde. 2010, sous la direction d'Alexandre Wolff, Paris, Éditions Nathan, p. 50-52.

BOUHDIBA, S. 2011. L'arabe et le français dans le système éducatif tunisien : approche démographique et essai prospectif. Québec: Observatoire démographique et statistique de l'espace francophone, Université Laval, 46 p.

Gouvernement du Québec, 2018, Partout, la culture. Politique culturelle du Québec, Culture et communications Québec, 63 p.

GRIN, F., 2010. «L'aménagement linguistique en Suisse », Télescope, 16 (3), 55-74.

GUIMOND, E. et SENÉCAl, S. 2009. «Les populations autochtones. Présentation », Cahiers québécois de démographie, $38(2), 217-220$.

HOULE, R et A. CAMBRON-PRÉMONT, A. 2015. Les concepts et les questions posées sur les langues aux recensements canadiens de 1901 à 1961. Cahiers québécois de démographie, 44(2), 291-310.

HUMBERT, P., R. CORAY et A. DUCHÊNE. 2018. Compter les langues : histoire, méthodes et politiques des recensements de la population. Une revue de la littérature. Université de Fribourg, Rapport du Centre scientifique des compétences sur Le plurilinguisme, 73 p. 
ISSAKA MAGA H. 2014. Alphabétisation et dynamique des langues au Niger: que nous apprennent les données censitaires et administratives? Québec: Observatoire démographique et statistique de l'espace francophone / Université Laval, $72 \mathrm{p}$.

KONATÉ, M.K., I. DIABATÉ et A. ASSIMA. 2010. Dynamique des langues locales et de la langue française au Mali : un éclairage à travers les recensements généraux de la population (1987 et 1998). Québec: Observatoire démographique et statistique de l'espace francophone / Université Laval, $46 \mathrm{p}$.

LACHAPELLE, R. 2008. "L'information démolinguistique et les définitions de " francophone » à des fins statistiques au Canada ", Séminaire international sur la méthodologie d'observation de la langue française dans le monde, AUF-OIF, Paris (juin 2008), p. 163-178.

MAHEU, R. 1985. "La démolingusitique : objet et enjeux », Cahiers québécois de démographie, volume 14, no 1, p. 3-5.

MARCOUX, R. 2018. "La place de l'Afrique dans la Francophonie : une question de nombres? ", Questions Internationales, no 90, La documentation française, (Thématique : «La nouvelle Afrique »), p. 113-117.

MARCOUX, R. avec la collaboration de M. GAGNÉ (2003), « La Francophonie de demain : essai de mesure de la population appartenant à la Francophonie d'ici 2050 », Cahiers québécois de démographie, vol. 32, no 2, p. 273-294.

MARCOUX, R. et A. WOLFF (2014). "Les tendances démographiques de quelques espaces linguistiques définis à partir de la langue officielle : 1960-2060 ", Une Francophonie en quête de sens. Retour sur le $1^{\text {er }}$ Forum mondial de la langue française, PUL, Québec, p. 35-46.

MARCOUX, R. et J. ALLADATIN (2014). « Les francophones analphabètes en Afrique : un phénomène relativement marginal », La langue française dans le monde. 2014, Éditions Nathan, Paris, p. 28-31.

MARCOUX, R., L. RICHARD et M. K. KONATÉ (2013). "Digital Preservation of Demographic Heritage. Population Censuses and Experiences in Mali and the Democratic Republic of the Congo ", The Memory of the World in the Digital Age: Digitization and Preservation, sous la direction de Luciana Duranti et Elizabeth Shaffer, UNESCO, p. 672-684.

MARCOUX, R. et L. RICHARD (2017). Tendances démographiques dans l'espace francophone. Québec, Observatoire démographique et statistique de l'espace francophone / Université Laval, $31 \mathrm{p}$.

MARCOUX, R. et M.K. KONATE (2012). "Africa and the Francophonie of Tomorrow : Attempt to Measure the Population of the Francophonie from Now to 2060 ", African Population Studies, volume 25, no 2, p. 215-225.

MARCOUX, R. et M.K. KONATÉ, (2008), « Les sources de données démolinguistiques en Afrique francophone ", Séminaire international sur la méthodologie d'observation de la langue française dans le monde, AUF-OIF, Paris (juin 2008), p. 351-367.

MAURER, B. 2015. Mesurer la francophonie et identifier les francophones. Inventaire critique des sources et des méthodes, Paris, Éditions des archives contemporaines, $229 \mathrm{p}$.

OUÉDRAOGO, A. E. et R. MARCOUX (2014). "Cohabitation des langues dans l'espace francophone : les exemples de 5 pays africains ", La langue française dans le monde. 2014, Éditions Nathan, Paris, p. 82-89. 
OUSMANE IDA, I. 2015. L'aphabétisation au Niger : une analyse à partir des données du recensement de 2012. Québec: Observatoire démographique et statistique de l'espace francophone / Université Laval, $44 \mathrm{p}$.

PICHÉ, V. 2011. "Catégories ethniques et linguistiques au Québec : quand compter est une question de survie ». Cahiers québécois de démographie, 40(1), 139-154.

PRÉVOST, J.G. et J-P. BEAUD, 2002. "Statistical Inquiry and the Management of Linguistic Plurality in Canada, Belgium and Switzerland", Journal of Canadian Studies, Volume 36, no 4, p. 88-117.

SIOUI-DURAND, G. 2018. "Sur les langues autochtones, Iwa'ah, yatàtiatha'endat, 'Je parle un peu Wendat" ", Recherches sociographiques, LIX, 1-2, p. 267-276.

TANANG TCHOUALA, P. et H.J. EFON ETINZOH. 2013. Les dynamiques démolinguistiques au Cameroun de 1960 à 2005 : un éclairage à travers les données des recensements. Québec: Observatoire démographique et statistique de l'espace francophone / Université Laval, 100 p.

TERMOTE, M. 2015. "Réflexions sur l'évolution du discours démolinguistique de Jacques Henripin ». Cahiers québécois de démographie, 44(2), 203-222.

UWAYEZU, B. 2015. Portrait démolinguistique du Rwanda: une analyse à partir des données des deux derniers recensements ( $R G P H 2002$ et RGPH 2012). Québec: Observatoire démographique et statistique de l'espace francophone / Université Laval, 42 p. 